\title{
Usage du mélange de la pepsine avec des préparations "Fromase 》 ou " Milcozyme » pour la fabrication des fromages Tilsit et Camembert
}

\author{
par \\ JARMUL (I)*, REPS (A)*, POZNANSKI (S.)*
}

\section{INTRODUCTION}

Pour faire coaguler le lait au cours de la fabrication de fromage, la pepsine était employée jusqu'à ce temps pour la plupart en mélange avec la présure. La présure faisant défaut, de plus en plus sensiblement, il est devenu nécessaire de la remplacer par son succédané d'origine microbienne.

Nos études précédentes [5] ont démontré que le mélange de la pepsine de porc avec la préparation "Fromase " qui est obtenue de la moisissure Mucor miehei, peut être employée, en proportion de $50 / 50$, ou $70 / 30$ pour la fabrication du fromage Edam, et, en proportion de $80 / 15$, pour la fabrication du fromage Kortowski (fromage polonais à pâte demi-molle).

On sait que l'activité protéolytique de la «Fromase » approche de celle de la présure $[10,13]$ et le fromage qui avait été fabriqué avec cette préparation, seule ou en mélange avec la pepsine, s'est montré l'emporter, par sa qualité, sur le fromage à présure $[1,5,6,10]$.

Les résultats favorables de nos essais nous ont inclinés à apprécier l'utilité de la pepsine de porc, en mélange avec les préparations "Fromase " ou " Milcozyme " pour la fabrication des fromages Tilsit et Camembert. La préparation "Milcozyme " est produite au Japon à l'aide de la bactérie Bacillus polymyxa et elle n'a pas été généralement admise pour la fabrication de fromage à cause de son activité protéolytique élevée $[4,8]$.

* Institut du Génie et de la Biotechnologie Alimentaire de l'Université Agrotechnique d'Olsztyn (Pologne). 


\section{PROTOCOLE EXPERIMENTAL}

Des fromages Tilsit et Camembert étaient fabriqués à l'échelle industrielle par des usines laitières spécialisées dans la fabrication de ces sortes de fromage.

La coagulation des protéines était effectuée à l'aide de :

- la présure en solution, à l'activité de coagulation de 1: 10000 ;

- la pepsine, à l'activité protéolytique de 1:4000 ;

- du mélange de la pepsine avec la préparation «Fromase » (activité de coagulation de $1: 100000$ ) ou avec la préparation "Milcozyme » (activité de coagulation de 1:80000).

Dans les mélanges de préparations utilisés, le taux de la pepsine était de 85,70 et 50 p. 100 et celui des succédanés microbiens de 15, 30 et 50 p. 100 calculés en activité de coagulation.

Les fromages expérimentaux, ainsi que les fromages témoins (fabriqués à l'aide de la présure et la pepsine seule) étaient affinés dans les conditions convenables à la sorte de fromage en question.

Au cours de l'affinage, les fromages étaient soumis à l'examen organoleptique par une commission d'experts. En même temps, le degré et la nature de la dégradation de protéines étaient étudiés, en déterminant la teneur en azote précipitable avec des ions calcium [9], celle en azote soluble à $\mathrm{pH}$ 4,6 [16], en azote non protéique [14] et peptidique [2], en acides aminés [17]. Des protéines de fromage étaient aussi séparées sur le gel Sephadex G-100 [15, 18] et par électrophorèse sur le gel de polyacrylamide $[3,7]$.

\section{PRESENTATION DES RESULTATS ET DISCUSSION}

On a constaté que les fromages de type Tilsit et Camembert peuvent être fabriqués, sans modifier des paramètres technologiques usuels, en faisant coaguler le lait à l'aide du mélange de la pepsine avec des succédanés de présure, " Fromase » ou " Milcozyme ».

Pendant la fabrication de ces fromages, aucune différence n'était constatée au cours de la formation du caillé à l'aide de la présure de la pepsine ou du mélange de la pepsine avec la "Fromase ».

En ce dernier cas, la caillé a été obtenu, avec une fermeté identique à celle du caillé à présure.

D'autre part, quelques différences ont été observées dans la coagulation du lait, les fromages étudiés étant fabriqués avec le mélange de la pepsine et du "Milcozyme ». Le commencement de la phase de coagulation était remarqué après $1 / 3$ du temps qui était nécessaire pour obtenir la fermeté du caillé convenable au fromage 
en question. De même, la fermeté du caillé, qui était obtenu avec le mélange pepsine - "Milcozyme » s'est montrée inférieure à celle qui était obtenue à l'aide de la présure, ou du mélange pepsine "Fromase ». La fermeté du caillé diminuait quand le taux du " Milcozyme » augmentait dans le mélange. Ces observations confirment nos études précédentes [11, 12].

Malgré des différences de la fermeté du caillé, qui était obtenu à l'aide des préparations étudiées, aucune différence n'était constatée au cours du traitement du caillé et des grains.

En analysant le degré d'utilisation des substances azotées de lait (tab. 1), il apparaît que la teneur du lactosérum en substances azotées était moins élevée au cours de la fabrication des fromages avec la pepsine, qu'elle ne l'était au cours de la fabrication du fromage à la présure.

Parmi les mélanges étudiés, ce sont des mélanges de la pepsine avec la "Fromase " à $70 / 30$ et à $50 / 50$, ou bien le mélange pepsine « Milcozyme » à $85 / 15$, qui ont permis d'obtenir le degré d'utilisation des substances azotées de lait approchant de celui de la présure. Dans la mesure où la quantité du "Milcozyme " augmentait dans le mélange avec la pepsine, le taux des substances azotées, qui passaient du lait au lactosérum, augmentait aussi sensiblement. Pour le mélange

\section{TABLEAU 1}

Taux de matières azotées passées du lait au lactosérum au cours de la fabrication du fromage

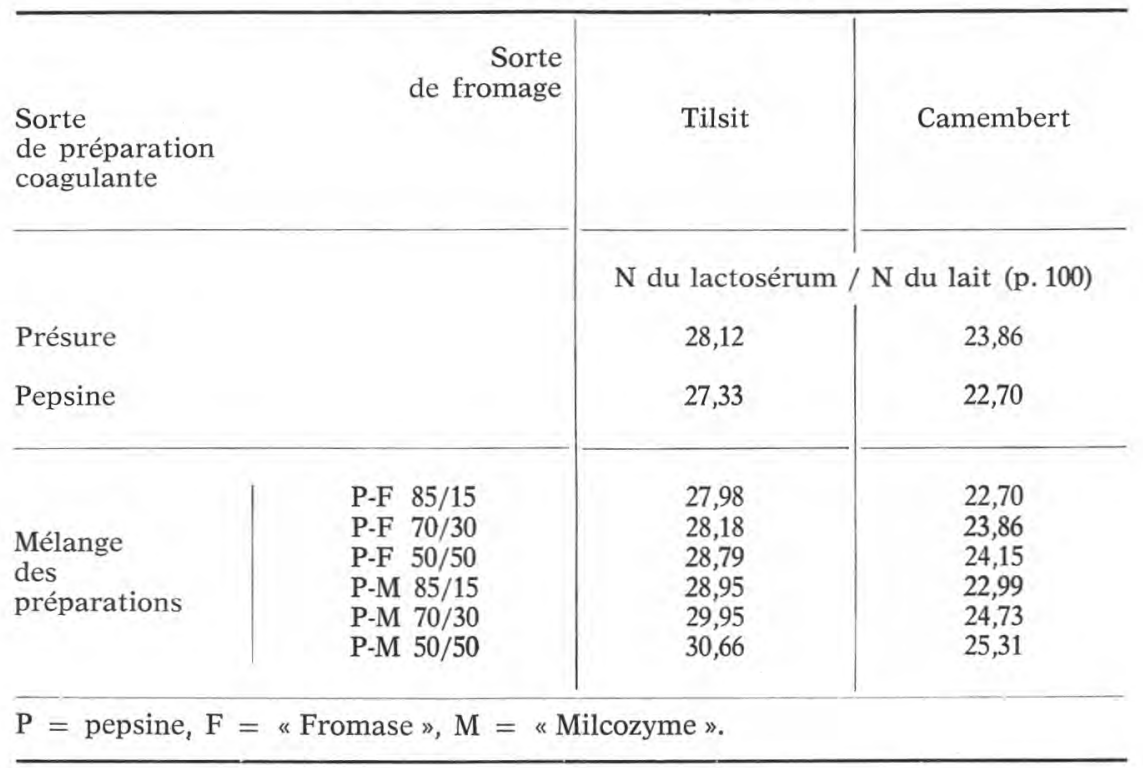


TABLEAU 2

Degré de la dégradation des protéines au cours de l'affinage du fromage

\begin{tabular}{|c|c|c|c|c|c|c|c|c|c|c|c|c|c|c|c|c|c|c|c|c|c|c|}
\hline \multirow{2}{*}{$\begin{array}{c}\text { Sorte } \\
\text { de } \\
\text { fromage }\end{array}$} & \multirow{2}{*}{\multicolumn{2}{|c|}{$\begin{array}{l}\text { Sorte de } \\
\text { préparation } \\
\text { coagulante }\end{array}$}} & \multicolumn{4}{|c|}{$\mathrm{N}$ précipité avec $\mathrm{Ca}^{++}$} & \multicolumn{4}{|c|}{$\mathrm{N}$ soluble à $\mathrm{pH} 4,6$} & \multicolumn{4}{|c|}{$\mathrm{N}$ non protéique } & \multicolumn{4}{|c|}{$\mathrm{N}$ de peptides } & \multicolumn{4}{|c|}{ N d'acides aminés } \\
\hline & & & \multicolumn{20}{|c|}{ en pourcentage de $\mathrm{N}$ total } \\
\hline Durée & $\begin{array}{l}\text { d'affin } \\
\text { semaine }\end{array}$ & $\begin{array}{l}\text { aage en } \\
\text { es }\end{array}$ & 2 & 4 & 8 & 12 & 2 & 4 & 8 & 12 & 2 & 4 & 8 & 12 & 2 & 4 & 8 & 12 & 2 & 4 & 8 & 12 \\
\hline \multirow[b]{2}{*}{ 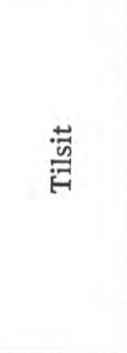 } & $\begin{array}{l}\text { Présur } \\
\text { Pepsin }\end{array}$ & & - & $\begin{array}{l}86,89 \\
88,89\end{array}$ & $\begin{array}{l}74,43 \\
81,42\end{array}$ & $\begin{array}{l}70,44 \\
75,23\end{array}$ & - & $\begin{array}{l}13,50 \\
11,72\end{array}$ & $\begin{array}{l}23,45 \\
18,60\end{array}$ & $\begin{array}{l}28,81 \\
23,34\end{array}$ & $\overline{-}$ & $\begin{array}{l}7,29 \\
6,31\end{array}$ & $\begin{array}{l}15,77 \\
10,97\end{array}$ & $\begin{array}{l}18,01 \\
14,99\end{array}$ & - & $\begin{array}{l}2,36 \\
1,89\end{array}$ & $\begin{array}{l}5,42 \\
3,07\end{array}$ & $\begin{array}{l}7,56 \\
3,99\end{array}$ & $\overline{-}$ & $\begin{array}{l}4,67 \\
5,02\end{array}$ & $\begin{array}{l}7,22 \\
7,05\end{array}$ & $\begin{array}{l}9,22 \\
8,39\end{array}$ \\
\hline & 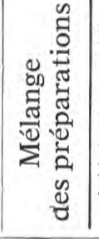 & $\begin{array}{ll}\text { P-F } & 85 / 15 \\
\text { P-F } & 70 / 30 \\
\text { P-F } & 50 / 50 \\
\text { P-M } & 85 / 15 \\
\text { P-M } & 70 / 30 \\
\text { P-M } & 50 / 50\end{array}$ & $\begin{array}{l}z \\
- \\
z \\
z\end{array}$ & $\begin{array}{l}87,76 \\
87,91 \\
87,04 \\
86,20 \\
85,20 \\
84,09\end{array}$ & $\begin{array}{l}78,55 \\
75,96 \\
75,15 \\
75,27 \\
74,97 \\
72,52\end{array}$ & $\begin{array}{l}73,46 \\
72,10 \\
70,72 \\
72,93 \\
72,14 \\
68,02\end{array}$ & $\begin{array}{l}z \\
z \\
z \\
-\end{array}$ & $\begin{array}{l}11,94 \\
12,20 \\
13,15 \\
13,30 \\
14,42 \\
15,85\end{array}$ & $\begin{array}{l}21,49 \\
23,35 \\
24,91 \\
23,26 \\
25,56 \\
27,11\end{array}$ & $\begin{array}{l}25,36 \\
26,96 \\
28,82 \\
27,04 \\
27,91 \\
30,79\end{array}$ & $\begin{array}{l}- \\
- \\
z \\
-\end{array}$ & $\begin{array}{l}5,95 \\
6,66 \\
7,01 \\
7,24 \\
7,30 \\
8,59\end{array}$ & $\begin{array}{l}11,71 \\
11,67 \\
12,93 \\
14,29 \\
17,35 \\
18,14\end{array}$ & $\begin{array}{l}15,07 \\
16,26 \\
17,11 \\
18,27 \\
18,83 \\
22,71\end{array}$ & $\begin{array}{l}- \\
- \\
- \\
-\end{array}$ & $\begin{array}{l}1,62 \\
1,47 \\
2,12 \\
1,96 \\
2,79 \\
2,96\end{array}$ & $\begin{array}{l}3,08 \\
3,21 \\
3,38 \\
3,72 \\
5,60 \\
6,53\end{array}$ & $\begin{array}{l}3,15 \\
3,37 \\
3,45 \\
5,45 \\
6,41 \\
7,28\end{array}$ & $\begin{array}{l}- \\
\overline{-} \\
\overline{-} \\
-\end{array}$ & $\begin{array}{l}5,03 \\
5,03 \\
4,40 \\
4,20 \\
4,58 \\
4,45\end{array}$ & $\begin{array}{l}8,03 \\
8,84 \\
8,96 \\
7,38 \\
9,32 \\
9,50\end{array}$ & $\begin{array}{l}8,46 \\
9,12 \\
9,95 \\
9,09 \\
9,21 \\
9,79\end{array}$ \\
\hline \multirow{2}{*}{$\begin{array}{l}\vec{U} \\
\text { : } \\
\text { हूँ } \\
\text { हूँ }\end{array}$} & \multicolumn{2}{|c|}{$\begin{array}{l}\text { Présure } \\
\text { Pepsine }\end{array}$} & $\begin{array}{l}71,07 \\
71,59\end{array}$ & $\overline{-}$ & $\overline{-}$ & $\overline{-}$ & $\begin{array}{l}28,55 \\
27,98\end{array}$ & $\overline{-}$ & $\overline{-}$ & $\overline{-}$ & $\begin{array}{l}20,46 \\
19,44\end{array}$ & $\overline{-}$ & $\overline{-}$ & - & $\begin{array}{l}5,68 \\
5,12\end{array}$ & $\overline{-}$ & $\overline{-}$ & $\overline{-}$ & $\begin{array}{l}8,40 \\
7,19\end{array}$ & $\overline{-}$ & $\overline{-}$ & $\overline{-}$ \\
\hline & 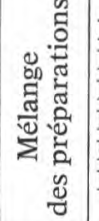 & $\begin{array}{ll}\text { P-F } & 85 / 15 \\
\text { P-F } & 70 / 30 \\
\text { P-F } & 50 / 50 \\
\text { P-M } & 85 / 15 \\
\text { P-M } & 70 / 30 \\
\text { P-M } & 50 / 50\end{array}$ & $\begin{array}{l}67,24 \\
66,67 \\
66,75 \\
66,63 \\
64,48 \\
63,53\end{array}$ & $\begin{array}{l}- \\
- \\
- \\
-\end{array}$ & $\begin{array}{l}- \\
\bar{z} \\
- \\
-\end{array}$ & $\begin{array}{l}\bar{z} \\
\overline{-} \\
\overline{-}\end{array}$ & $\begin{array}{l}29,77 \\
29,90 \\
29,79 \\
31,11 \\
34,47 \\
34,86\end{array}$ & $\begin{array}{l}z \\
\overline{-} \\
\overline{-} \\
-\end{array}$ & $\begin{array}{l}- \\
- \\
- \\
-\end{array}$ & $\begin{array}{l}\bar{z} \\
\overline{-} \\
\overline{-}\end{array}$ & $\begin{array}{l}20,07 \\
20,44 \\
20,28 \\
20,27 \\
22,07 \\
22,11\end{array}$ & $\begin{array}{l}- \\
- \\
- \\
-\end{array}$ & $\begin{array}{l}z \\
z \\
z \\
-\end{array}$ & $\begin{array}{l}- \\
\overline{-} \\
- \\
-\end{array}$ & $\begin{array}{l}5,47 \\
5,21 \\
5,40 \\
5,76 \\
5,71 \\
5,72\end{array}$ & $\begin{array}{l}z \\
- \\
- \\
-\end{array}$ & $\begin{array}{l}- \\
\overline{-} \\
\overline{-} \\
-\end{array}$ & $\begin{array}{l}\overline{-} \\
\overline{-} \\
\overline{-}\end{array}$ & $\begin{array}{l}7,78 \\
8,57 \\
8,40 \\
7,90 \\
8,70 \\
8,60\end{array}$ & $\begin{array}{l}- \\
z \\
- \\
-\end{array}$ & $\begin{array}{l}\overline{-} \\
\overline{-} \\
\overline{-}\end{array}$ & $\begin{array}{l}- \\
- \\
- \\
-\end{array}$ \\
\hline
\end{tabular}

$\mathrm{P}=$ pepsine, $\mathrm{F}=$ «Fromase », $\mathrm{M}=$ « Milcozyme ». 
pepsine - "Milcozyme " à 50/50, ce taux était plus élevé que le taux des substances azotées qui passaient au lactosérum au cours de la fabrication du fromage à présure. L'augmentation était de 9 p. 100 $(30,66$ vis-à-vis du 28,12 p. 100$)$ pour le fromage Tilsit et de 6 p. 100 $(25,31$ vis-à-vis de 23,86 p. 100) pour le Camembert.

L'analyse de la teneur en formes diverses des combinaisons d'azote (tab. 2) a confirmé les résultats de nos études des précédantes, à savoir que le fromage fabriqué avec la pepsine s'affinait plus lentement que le fromage à la présure. Dans les fromages analysés, fabriqués à l'aide de la pepsine en mélange avec la "Fromase " ou le "Milcozyme », le degré de protéolyse augmente à mesure que le taux de la préparation microbienne augmente dans le mélange et particulièrement dans le cas de la préparation " Milcozyme ».

L'étendue de la protéolyse qui approchait le plus de celle du fromage à la présure, a été constatée dans le cas du fromage Tilsit qui était fabriqué avec les mélanges pepsine- «Fromase » à 50/50 et pepsine - "Milcozyme » à 85/15. Pour le fromage Camembert les proportions les plus favorables étaient : le mélange pepsine - "Fromase » à 85/15 et le mélange pepsine - "Milcozyme » à 85/15.

La séparation chromatographique des protéines du fromage Tilsit sur le gel Sephadex G-100 (tab. 3) a montré que le caractère de la dégradation des protéines dépendait du type de mélange utilisé.

Après la période d'affinage, exigée par la norme de qualité, la présence de quatre fractions de protéines était constatée dans le fromage à présure, deux fractions dans le fromage à la pepsine et dans celui qui était fabriqué avec le mélange pepsine - "Fromase ", et cinq fractions dans le fromage fabriqué avec le mélange pepsine"Milcozyme » à 50/50. Dans le fromage qui était fabriqué avec le mélange pepsine - "Fromase », le degré de dégradation des protéines était semblable à celui du fromage à la pepsine.

De même que dans les fromages Edam et Kortowski, qui étaient fabriqués à l'aide de pepsine et son mélange avec la "Fromase » [5], dans le fromage Tilsit, le pourcentage de protéines au poids moléculaire le plus élevé était supérieur à celui du fromage à présure.

En employant le mélange pepsine - "Milcozyme » pour la fabrication du fromage Tilsit, on a constaté que l'intensité de la dégradation des protéines qui était provoquée par ce mélange, était plus élevée que dans le cas des autres préparations coagulantes qui sont en usage pour la production de ces fromages.

Par exemple, après 8 semaines d'affinage, le fromage qui était fabriqué avec le mélange pepsine- "Fromase» à 50/50 contenait 27,19 p. 100 des protéines au poids moléculaire le plus bas. La substitution partielle de 15 p. 100 de "Milcozyme » à la pepsine faisait augmenter le taux de ces protéines jusqu'à 30,49 p. 100 . La préparation "Milcozyme " augmentant dans le mélange, une réduction significative de la teneur en protéines de poids moléculaire le plus élevé était 
TABLEAU 3

Caractéristique de la répartition chromatographique des protéines du fromage Tilsit sur le Gel Séphadex G-100

\begin{tabular}{|c|c|c|c|c|c|c|c|c|c|}
\hline \multirow{2}{*}{\multicolumn{3}{|c|}{$\begin{array}{l}\text { Sorte de préparation } \\
\text { coagulante }\end{array}$}} & \multicolumn{4}{|c|}{ Présure } & \multicolumn{3}{|c|}{ Pepsine } \\
\hline & & & 1 & 3 & 4 & 5 & 1 & 4 & 5 \\
\hline \multirow[b]{2}{*}{$\subseteq \stackrel{8}{c}$} & \multirow{2}{*}{4} & $\begin{array}{rr}\text { Masse moléculaire } \\
\times 10^{-3} \\
\end{array}$ & 126 & 54 & - & 15 & 126 & 35 & 14 \\
\hline & & $\frac{N \text { du pic }}{N^{\prime} \text { elué }}[\%]$ & 58,85 & 15,47 & - & 2568 & 58,38 & 20,27 & 21,35 \\
\hline \multirow{2}{*}{ 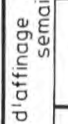 } & \multirow{2}{*}{8} & $\begin{array}{r}\text { Masse moléculaire } \\
\times 10^{-3}\end{array}$ & 126 & 47 & 32 & 15 & 126 & - & 13 \\
\hline & & $\frac{N}{N^{\prime}} \frac{\text { du pic }}{\text { elué }}[\%]$ & 50,83 & 8,12 & 6,07 & 34,98 & $71 / 48$ & - & 28, \\
\hline \multirow{2}{*}{ 选 } & \multirow{2}{*}{12} & $\begin{array}{r}\text { Masse moléculaire } \\
\times 10^{-3}\end{array}$ & 126 & 50 & 35 & 15 & 126 & - & 13 \\
\hline & & $\frac{N \text { du pic }}{N^{\prime} \text { elue }}[\%]$ & 36,58 & 6,92 & 5,25 & 51,27 & 69,96 & - & 0,04 \\
\hline
\end{tabular}

\begin{tabular}{|c|c|c|c|c|c|c|c|c|}
\hline \multicolumn{7}{|c|}{ Mélange des préparations } \\
\hline$P-F$ & $85 / 15$ & \multicolumn{2}{|c|}{$P$ - F } & $70 / 30$ & \multicolumn{3}{|c|}{$P-F$ 50/50 } \\
\hline 1 & 3 & 5 & 1 & 4 & 5 & 1 & 4 & 5 \\
\hline 126 & 47 & 15 & 126 & 47 & 14 & 126 & 47 & 15 \\
\hline 57,82 & 21,09 & 21,09 & 54,56 & 26,29 & 19,15 & 52,18 & 25,12 & 22,70 \\
\hline 126 & - & 15 & 126 & - & 14 & 126 & - & 15 \\
\hline 73,84 & - & 26,16 & 72,54 & - & 27,46 & 72,81 & - & 27,19 \\
\hline 126 & - & 15 & 126 & - & 14 & 126 & - & 15 \\
\hline 70,00 & - & 30,00 & 65,00 & - & 35,00 & 65,44 & - & 34,56 \\
\hline
\end{tabular}

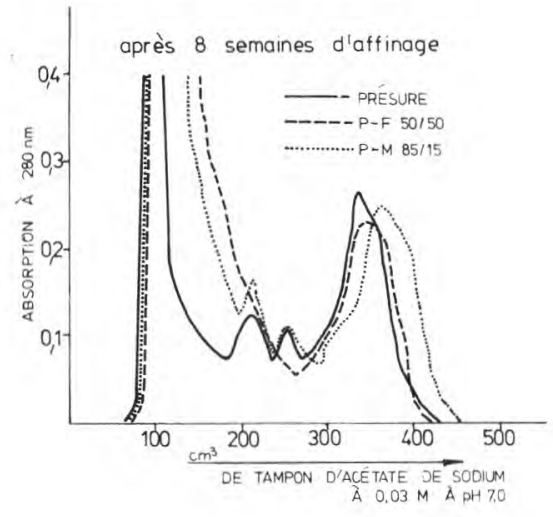

\begin{tabular}{|c|c|c|c|c|c|c|c|c|c|c|c|c|c|}
\hline \multicolumn{14}{|c|}{ Mélange des préparations } \\
\hline \multicolumn{5}{|c|}{$P-M \quad 85 / 15$} & \multicolumn{4}{|c|}{ P-M 70/30 } & \multicolumn{5}{|c|}{ P-M $50 / 50$} \\
\hline 1 & 2 & 3 & 4 & 5 & 1 & 3 & 4 & 5 & 1 & 2. & 3 & 4 & 5 \\
\hline 126 & 80 & 54 & - & 13 & 126 & 69 & 40 & 19 & 126 & 85 & 54 & 32 & 15 \\
\hline 37,85 & 17,00 & 17,26 & - & 27,89 & 21,75 & 19,97 & 17,09 & 41,19 & 8,06 & 9,20 & 8,12 & 23,54 & 5108 \\
\hline 126 & - & 47 & 32 & 13 & 126 & 54 & 35 & 15 & 126 & 80 & 47 & 37 & 12 \\
\hline 31,92 & - & 20,15 & 17,44 & 30,49 & 11,68 & 21,10 & 21,52 & 45,70 & 10,66 & 7,70 & 7,58 & 15,40 & 58,66 \\
\hline 126 & - & 54 & 32 & 13 & 126 & 54 & 32 & 15 & 126 & - & 43 & 25 & 12 \\
\hline 9,41 & - & 29,2 & 1048 & 0,91 & 10,13 & 10,48 & 20,41 & 58,98 & 7,46 & - & 405 & 1261 & 75,8 \\
\hline
\end{tabular}

$\mathrm{P}=$ pepsine, $\mathrm{F}=$ "Fromase », $\mathrm{M}=$ " Milcozyme ». 


\section{TABLEAU 4}

Caractéristique de la répartition chromatographique des protéines du fromage Camembert sur le gel Séphadex G-100 après 2 semaines d'affinage

\begin{tabular}{|c|c|c|c|c|}
\hline $\begin{array}{l}\text { Sorte de préparation } \\
\text { coagulante }\end{array}$ & \multicolumn{2}{|c|}{ Présure } & \multicolumn{2}{|c|}{ Pepsine } \\
\hline No du pic & 1 & 2 & 1 & 2 \\
\hline $\begin{array}{r}\text { Masse moléculaire } \\
\times 10^{-3}\end{array}$ & 126 & 13 & 126 & 13 \\
\hline$\frac{N \text { du pic }}{N^{\prime} \text { elué }}[\%]$ & 9,71 & 90,29 & 8,79 & 91,21 \\
\hline
\end{tabular}

\begin{tabular}{|c|c|c|c|c|c|}
\hline \multicolumn{5}{|c|}{ Mélange des préparations } \\
\hline P-F $85 / 15$ & P-F & $70 / 30$ & \multicolumn{2}{|c|}{ P-F $50 / 50$} \\
\hline 1 & 2 & 1 & 2 & 1 & 2 \\
\hline 108 & 13 & 108 & 13 & 108 & 13 \\
\hline 5,27 & 94,73 & 5,01 & 94,99 & 5,75 & 94,25 \\
\hline
\end{tabular}

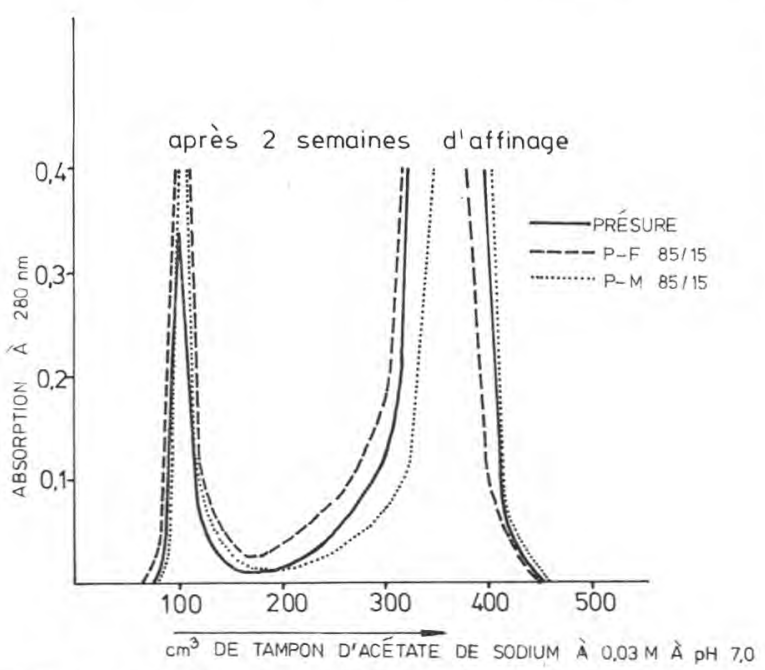

\begin{tabular}{|c|c|c|c|c|c|}
\hline \multicolumn{5}{|c|}{ Mélange des préparations } \\
\hline P-M $85 / 15$ & P-M & $70 / 30$ & P-M & $50 / 50$ \\
\hline 1 & 2 & 1 & 2 & 1 & 2 \\
\hline 108 & 13 & 108 & 12 & 108 & 12 \\
\hline 5,70 & 94,30 & 6,54 & 93,46 & 6,77 & 93,23 \\
\hline
\end{tabular}

$\mathrm{P}=$ pepsine, $\mathrm{F}=$ "Fromase, $\mathrm{M}=$ " Milcozyme».

observée dans le fromage. En même temps, la quantité de protéines de poids moléculaire le plus bas augmentait.

L'analyse de la séparation chromatographique du fromage Camembert (tab. 4.) a montré que la dégradation des protéines y procédait d'une manière semblable. Dans le fromage témoin, ainsi que dans le fromage expérimental, la présence de deux fractions de protéines était constatée, dont les pourcentages étaient identiques.

Des séparations électrophorétiques de protéines des fromages analysés qui étaient effectuées sur le gel de polyacrylamide, ont confirmé l'activité protéolytique supérieure de la présure, vis-à-vis de la pepsine. Il n'a pas été observé de différences essentielles dans la nature de la dégradation des protéines. Cependant, dans le fromage Tilsit à la présure, âgé de 12 semaines (fig. 1) et dans le fromage 


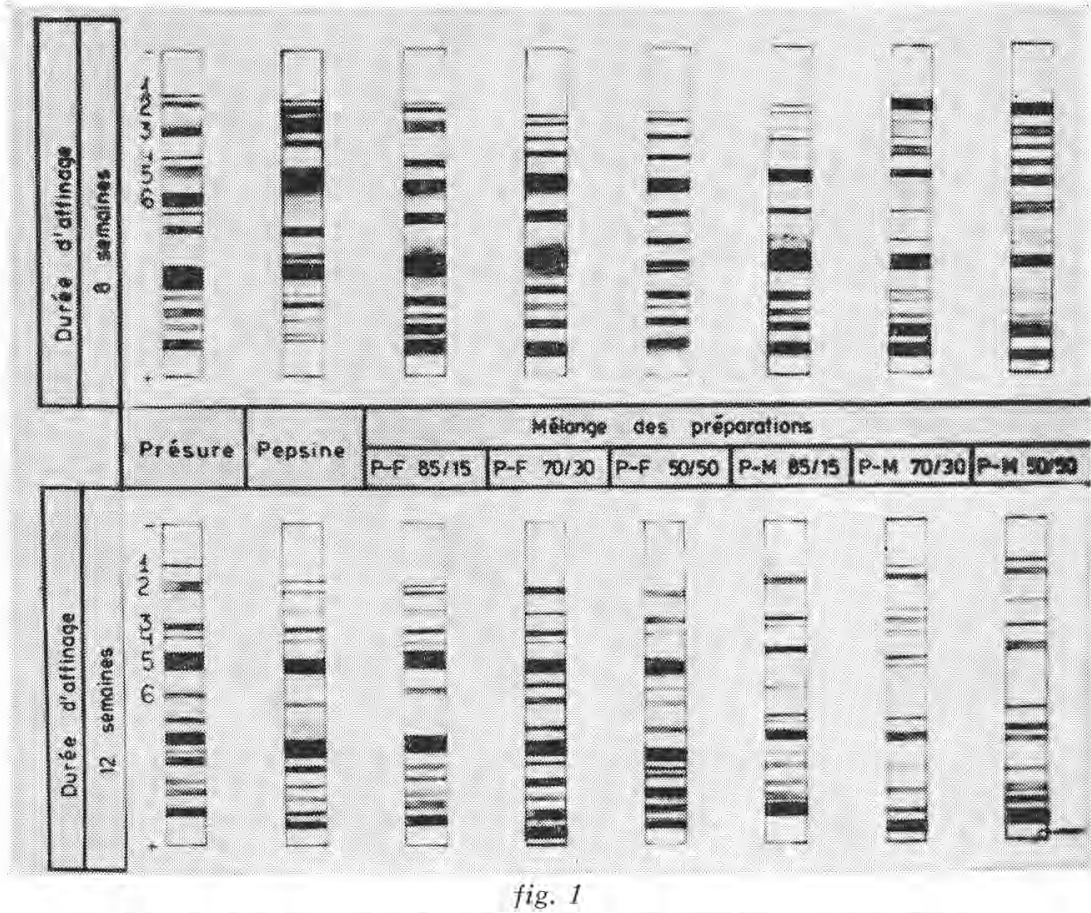

Répartition électrophorétique des protéines du fromage Tilsit sur le gel de polyacrylamide

$\mathrm{P}=$ pepsine, $\mathrm{F}=$ «Fromase, $\mathrm{M}=$ " Milcozyme ».

Camembert de 3 semaines (fig. 2) le pourcentage de la bande qui

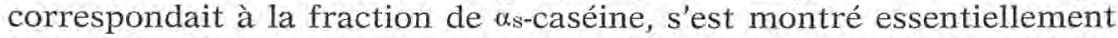
moins élevé. La dégradation plus lente des protéines du fromage Tilsit, qui était fabriqué à l'aide de la pepsine, est témoignée par le pourcentage de la bande qui correspondait à la fraction de $\beta$-caséine.

En analysant des séparations sur le gel de polyacrylamide, des protéines des fromages qui étaient fabriqués à l'aide du mélange pepsine - «Fromase » (fig. 1) on a constaté que la dégradation des protéines procédait plus lentement dans des fromages Tilsit, fabriqués avec les mélanges à $85 / 15$ et $70 / 30$ que dans le fromage à présure. Le procédé d'affinage un peu plus rapide, par comparaison avec le fromage à présure, était observé dans le fromage Tilsit fabriqué avec le mélange à 50/50. Le pourcentage de la bande de $\alpha_{s}+$ caséine $\beta$ y était moins élevé. D'autre part, le fromage Camembert (fig. 2) fabriqué avec le mélange pepsine - "Fromase » s'affinait un peu plus vite que le fromage à la présure. L'étendue de la protéolyse augmentait quand le taux de la "Fromase » augmentait dans le mélange avec la pepsine. 


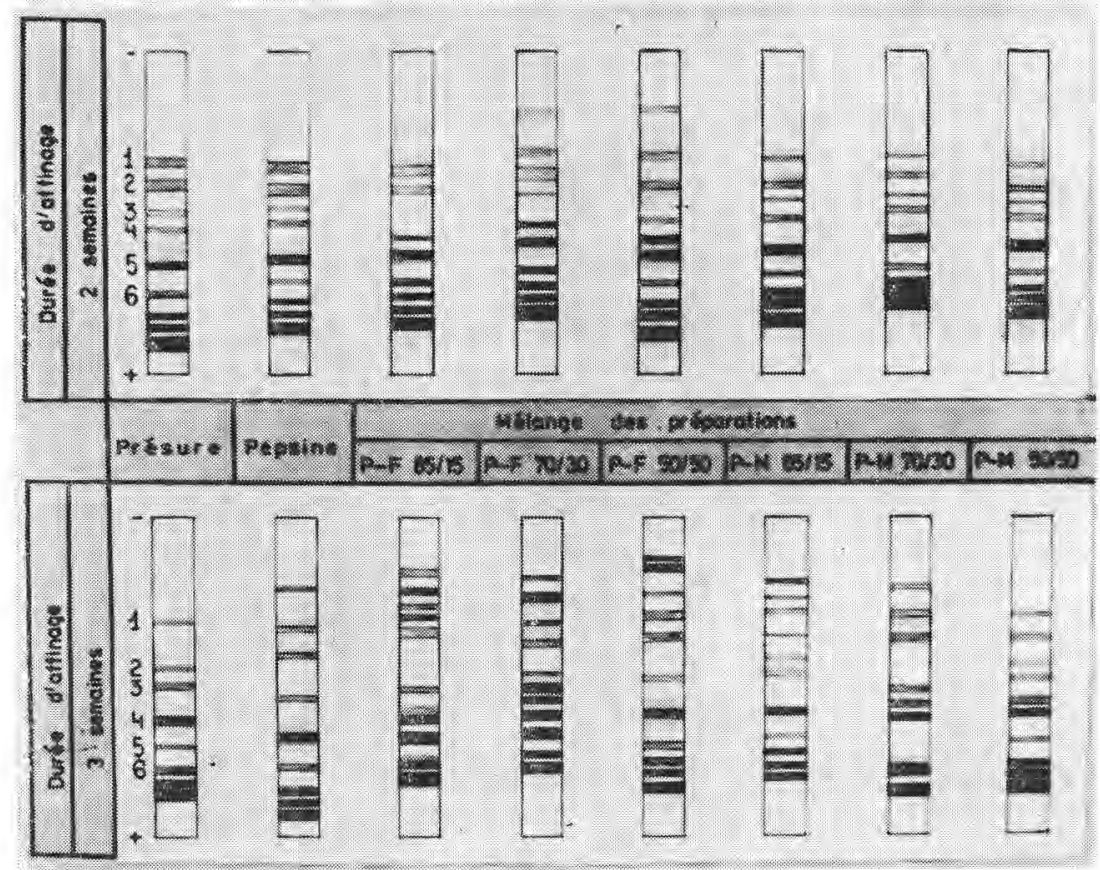

fig. 2

Répartition électrophorétique des protéines du fromage Camembert sur le gel de polyacrylamide

$\mathrm{P}=$ pepsine, $\mathrm{F}=$ «Fromase, $\mathrm{M}=$ « Milcozyme».

C'est d'une manière tout à fait différente que s'effectuait la dégradation des protéines dans le fromage qui était fabriqué avec le mélange pepsine - "Milcozyme» (fig. 1, 2). Dans ce fromage, la coloration de la bande, qui correspondait à $\alpha_{s}+$ caséine $\beta$, était moins intense, et elle s'affaiblissait quand le taux de la préparation augmentait dans le mélange, ce qui prouve que l'affinage de ce fromage procédait plus vite. La dégradation des protéines approchant le plus de celle du fromage à présure était observée dans le fromage qui était fabriqué à l'aide du mélange à 85/15.

Par contre, sur les électrophorogrammes des fromages Camembert, qui étaient fabriqués avec la présure, ou avec le mélange pepsine"Milcozyme» (fig. 2), on a observé le même nombre de fractions de protéine avec un pourcentage voisin. Après 3 semaines d'affinage le niveau de dégradation des protéines s'était montré plus intense dans le fromage avec le mélange pepsine - "Milcozyme».

$\mathrm{Au}$ cours de l'affinage de fromage, la fraction caséine $\alpha_{\mathbb{S}^{-}}$était đégradée par le mélange de pepsine avec les préparations microbiennes étudiées à un degré plus élevé, que ne l'était la fraction caséine $\beta$ par 
la présure. Pourtant, dans les fromages fabriqués avec le mélange de la pepsine et des préparations microbiennes étudiées, et surtout dans ceux avec le "Milcozyme ", la fraction caséine $\beta$ était dégradée d'une manière plus intense que dans les fromages à la présure.

L'examen organoleptique des fromages du type Tilsit et Camembert a démontré que les fromages qui étaient fabriqués à l'aide de la pepsine en mélange avec la «Fromase » l'emportaient sur les fromages à la présure par leur qualité .

Au cours de la fabrication des fromages avec le mélange de la pepsine avec la préparation "Milcozyme ", une qualité des fromages étudiée, supérieure à celle du fromage à la présure, était obtenue à l'aide du mélange pepsine - «Milcozyme » à $85 / 15$, tandis que dans les fromages avec les mélanges à $70 / 30$ et $50 / 50$, les défauts de goût, d'odeur et de consistance apparaissaient après la période prévue par la norme de qualité, ces défauts s'intensifiaient au cours de l'affinage.

Les résultats que nous avons obtenus indiquent que l'usage des mélanges pepsine - "Fromase » à $50 / 50$ ou pepsine- « Milcozyme » $85 / 15$ est le plus profitable pour la fabrication du fromage Tilsit, tandis que les mélanges pepsine - «Fromase » 85/15 et pepsine - " Milcozyme " $85 / 15$ sont préférables pour la fabrication du fromage Camembert.

\section{$R$ és u mé}

On a étudié l'utilité de la pepsine de porc, en mélange avec des préparations "Fromase " et "Milcozyme " pour la fabrication des fromages Tilsit et Camembert.

L'analyse a porté les paramètres technologiques de la fabrication de fromage, de l'utilisation des fractions azotées de lait, de la dégradation des protéines au cours de l'affinage du fromage.

On a constaté que l'usage des mélanges pepsine - "Fromase » à $50 / 50$ et pepsine - "Milcozyme » $85 / 15$ était préférable pour la fabrication du fromage Tilsit, tandis que pour le Camembert les mélanges où le taux d'une préparation microbienne est de 15 p. 100 étaient ceux à recommander.

\section{S u m m a ry}

APPLICATION OF PEPSIN IN MIXTURE WITH «FROMASE» OR «MILCOZYME» PREPARATION IN CHEESE-MAKING OF TILSIT AND CAMEMBERT

Studies were made on the suitability of porcine pepsin, as mixed with "Fromase» or "Milcozyme» preparations in the manufacture of Tilsit and Camembert cheeses.

Technical parameters of cheese ripening, utilization of nitrogen compounds as well as protein degradation were analysed in the course of cheese ripening. 
The mixtures of pepsin-«Fromase» 50/50 and pepsin- «Milcozyme» $85 / 15$ were found to be preferable for use in the Tilsit cheese manufacture, while for the Camembert cheese manufacture the mixtures containing $15 \%$ of either microbial preparation appeared to be convenient.

\section{Bibliographie}

[1] Antila (V.) und Witting (O.) (1976). - Der Einfluss von verschiedenen Labpräparaten auf die Reifung von Edamer Käse. Milchwiss., 31, 654.

[2] Boulanger (P.) et BiSerte (G.) (1949). - Chromatographie de partage des amino-acides libres du plasma sanguin. Bull. Soc. Chimie Biol., 31, 696.

[3] Davis (B. J.) (1964). - Disc electrophoresis. II. Method and application to human serum proteins. Ann. New York Acad. Sc., 121, 404.

[4] Iтон (T.) and Thomasow (J.) (1971). - Action of rennet and other milk clotting enzymes on casein fractions. Milchwiss., 26, 671.

[5] Jarmul (I.), Reps (A.), Poznanski (S.) et Zelazowska (H.) (1982). - Utilisation du mélange de la pepsine avec la préparation microbienne « Fromase " dans la fabrication des fromages Edam et Kortowski. Le Lait, $62,75-86$.

[6] MARTENS (R.) (1973). - Gouda cheese made with microbial rennets derived from Mucor miehei. Milchwiss., 28, 87.

[7] Ornstein (A. A.) (1964). - Disc electrophoresis. I. Background and theory. Ann. New York Acad. Sc., 121, 321.

[8] Poznanski (S.), Reps (A.), Kowalewska (J.), Rymaszewski (J.) and JedryCHOWSKI (L.) (1974). - Proteolytic activity of coagulating preparations of microbial origin depending on a kind of substrate. Milchwiss., 29, 742.

[9] Poznanski (S.) and Rymaszewski (J.) (1965). - Proteolysis during the ripening of Edam cheese with the preparation of some bacteria strains. I. Changes in particular nitrogen fractions. Milchwiss., 20, 14.

[10] REPS (A.) (1979), - Wlasciwosci substrtutow podpuszczki produkowanych przy uzyciu plesni Mucor miehei. Zesz. nauk. ART Olszt., 14, 271.

[11] Reps (A.), Poznanski (S.), Babuchowski (A.) et JedRychowsKi (L.) (1979), Propriétés des substituts de présure fabriqués à partir de Mucor miehei. Le Lait, 59, 1.

[12] Reps (A.), Poznanski (S.) and JakUbowski (J.) (1973). - Main properties of milk coagulating preparations of microbial origin. Milchwiss., 30, 65.

[13] Reps (A.), Poznanski (S.), Jedrychowski (L.), Zelazowska (H.) and BabucHowskI (A.) (1978). - Characterization of the microbial rennet Fromase. Milchwiss., 33, 34.

[14] Schober (R.), Niclaus (W.) und Christ (W.) (1961). - Anwendung der «Finger-Abdruck-Methode» auf die Kennzeichnung von Käsesorten durch ihre proteolytischen Inhaltsstoff. Milchwiss., 16, 140.

[15] Sephadex. Gel filtration in theorie and practice (1970), - Pharmacia Fine Chemicals.

[16] SODE-Mogensen (T.) (1948). - Determination of the degree proteolytic decomposition in cheese with special reference to the formal titration. Meddelande, No 21, Fran Statens Mejeriförsök, Alnarp-Akarp.

[17] Stadhouders (J.) (1960), - The hydrolysis of protein during the ripening of dutch cheese. The enzyme and bacteria involved. Neth. Milk Dairy I., 14,83

[18] ToKITA (F.) and Hosono (A.) (1968). - An investigation about Limburger cheese ripening by gel filtration on Sephadex. Milchwiss., 23, 758. 